\title{
A banalização da prescrição de psicofármacos em um ambulatório de saúde mental ${ }^{1}$
}

\author{
Daniele de Andrade Ferrazza ${ }^{2}$ \\ Cristina Amélia Luzio \\ Luiz Carlos da Rocha \\ Raphael Rodrigues Sanches \\ Universidade Estadual Paulista Júlio Mesquita Filho, Assis-SP, Brasil
}

\begin{abstract}
Resumo: A conjunção do desenvolvimento dos psicofármacos modernos com o amplo alcance que assumiu a ênfase preventiva em saúde mental na atualidade modificou as práticas da Psiquiatria, que deixou de ser um saber voltado exclusivamente ao tratamento da loucura para dedicar-se a medicar, através da prescrição de psicofármacos, qualquer manifestação de sofrimento psíquico. O presente trabalho problematiza esse processo atual de medicalização generalizada da população e apresenta dados de um estudo exploratório amostral que objetivou conhecer, através do exame de registros de prontuários, a trajetória dos usuários da porta de entrada do Pronto Atendimento até a prescrição de psicofármacos no âmbito de um Ambulatório de Saúde Mental de uma pequena cidade do oeste paulista. Nossos dados mostram que a maioria (65\%) dos usuários já chega ao serviço sob prescrição prévia de psicofármacos e que, encaminhados à consulta psiquiátrica, praticamente todos $(99 \%)$ recebem prescrição de psicofármacos.
\end{abstract}

Palavras-chave: saúde mental, saúde pública, farmacoterapia.

\section{Trivializing the prescription of psychopharmacologic drugs in a mental health service}

\begin{abstract}
The development of modern psychiatric medications coupled with the wide range currently gained by preventive emphasis in mental health changed the practices of psychiatry, which is no longer focused on treating insanity but is also dedicated to treat any psychiatric suffering through the prescription of psychopharmacos. This study discusses the current process in which medication has been generalized, and presents the results of an exploratory study aimed to examine the patients' medical files, the trajectory of users since they enter the service to the prescription of psychiatric medication in the scope of a Mental Health Outpatient Clinic, in a town in the west of the São Paulo state, Brazil. Results revealed that most $(65 \%)$ users already arrive at the service with previous prescription of psychiatric medications, and nearly all (99\%) of them receive prescriptions of psychiatric medication once forwarded to psychiatric consultations.
\end{abstract}

Keywords: mental health, public health, pharmacotherapy.

\section{La banalización de la prescripción de psicofármacos en un ambulatorio de salud mental}

\begin{abstract}
Resumen: La conjunción del desarrollo de los psicofármacos modernos con el amplio alcance que asumió la énfasis preventiva en salud mental en la actualidad modificó las prácticas de la psiquiatría, que dejó de ser un saber vuelto exclusivamente al tratamiento de la locura para dedicarse la medicar cualquier manifestación de sufrimiento psíquico. El presente trabajo problematiza ese proceso actual de medicalización generalizada y presenta datos de un estudio de investigación amostral que objetivó conocer, a través del examen de registros de lista medica, la trayectoria de los usuarios de la puerta de entrada de la Lista Atención hasta la prescripción de psicofármacos en el ámbito de un Ambulatorio de Salud Mental de una pequeña ciudad del oeste paulista. Nuestros datos muestran que la mayoría $(65 \%)$ de los usuarios ya llega al servicio bajo prescripción previa de psicofármacos y que, encaminados a la consulta psiquiátrica, prácticamente todos (99\%) reciben prescripción de psicofármacos.
\end{abstract}

Palabras clave: salud mental, salud publica, farmacoterapia.

Com a descoberta dos psicofármacos na década de $50 \mathrm{e}$ com ênfase preventiva que assumiu o atendimento psiquiátrico após a II Guerra Mundial, a psiquiatria modificou suas

\footnotetext{
${ }^{1}$ Apoio: FAPESP. Este trabalho é derivado da Dissertação de Mestrado defendida pela primeira autora, sob a orientação da segunda, no Programa de Pós-graduação em Psicologia da Faculdade de Ciências e Letras UNESP/Assis da Universidade Estadual Paulista Júlio Mesquita Filho.

${ }^{2}$ Endereço para correspondência:

Daniele de Andrade Ferrazza. Departamento de Psicologia Evolutiva, Social e Escolar. Avenida Dom Antônio, 2100. CEP: 19.806-900. AssisSP. E-mail: danieleferrazza@yahoo.com.br
}

práticas e deixou de ser um saber voltado exclusivamente ao tratamento da loucura para dedicar-se a medicar qualquer manifestação de sofrimento psíquico, chegando mesmo a recomendar a medicação de pessoas reconhecidamente portadoras de perfeita saúde mental (Gentil e cols., 2007).

Atualmente, qualquer sinal de sofrimento psíquico pode ser rotulado como uma patologia cujo tratamento será a administração de psicofármacos (Amarante, 2007; Barros, 2008; Birman, 2000; Ignácio \& Nardi, 2007; Lamb, 2008). Essa tendência tem-se ampliado de tal modo que se pode falar da ocorrência de uma generalizada "medicalização do social" (Birman, 2000). Sob esse prisma, os psicofármacos 
instituíram-se como o recurso terapêutico mais utilizado para tratar qualquer mal-estar das pessoas, em que se destaca a tristeza, o desamparo, a solidão, a inquietude, o receio, a insegurança, ou até mesmo a ausência de felicidade.

Por certo, aqui não se pretende discutir a específica adequação de diagnósticos ou particularidades técnicas da terapêutica medicamentosa, o que estaria fora de nossos objetivos e condições técnico-científicas. Muito diferente disso, o objeto geral de estudo desta pesquisa pode ser definido como o próprio processo de medicalizar, neologismo de significado distinto de medicar e aqui compreendido como fazer de algo ou alguém objeto do saber, da prática, ou da tutela médica que, na atualidade, apresenta o crescente processo de prescrição de psicofármacos como uma de suas principais manifestações. Bem por isso, escolhemos referenciar este estudo em compreensões críticas desse processo, amplamente presentes na bibliografia sobre o tema.

A noção de medicalização nos remete a um fenômeno complexo, polêmico e multifacetado. Conforme o verbete elaborado por Hora (2006) para o glossário do Grupo de Estudos e Pesquisas "História, Sociedade e Educação no Brasil" (HISTEDBR), medicalização pode ser compreendido como:

o processo pelo qual o modo de vida dos homens é apropriado pela medicina e que interfere na construção de conceitos, regras de higiene, normas de moral e costumes prescritos - sexuais, alimentares, de habitação - e de comportamentos sociais. Este processo está intimamente articulado à idéia de que não se pode separar o saber - produzido cientificamente em uma estrutura social - de suas propostas de intervenção na sociedade, de suas proposições políticas implícitas. A medicalização tem como objetivo a intervenção política no corpo social (s/p).

No mesmo verbete, como uma variante da noção de medicalização, a mesma autora situa a expressão "medicalização do social" como originária do estudo das sociedades disciplinares realizados por Foucault (1979; 2006), compreendendo-a como:

A forma pela qual a evolução tecnológica vem modificando a prática da medicina por meio de inovações dos métodos de diagnóstico e terapêutico, da indústria farmacêutica e de equipamentos médicos; por outro lado, pode ser usado numa referência às conseqüências que acarreta para o jogo de interesses envolvidos na produção do ato médico (Hora, 2006, s/p).

O saber médico, compreendido como o domínio de saber que historicamente vem abarcando toda a amplitude dos problemas da saúde humana, se materializa em práticas discursivas específicas que têm definido o que é normal, o que é próprio, o que é tratamento. Conforme a apreciação crítica de Cordeiro (1985), essa tendência normativa da medicina vem exercendo sobre a população uma particular forma de controle social que atinge "todas as dimensões da vida do indivíduo, ao legitimar e institucionalizar o papel do doente" (p. 38). Esse desdobramento político da prática médica é comentado por França (1994, p. 50):

Nesse fato político de gerenciar a vida humana, a medicina adquire um papel normativo e pedagógico que autoriza a uma ação permanente no corpo social. Distribuir conselhos, reger relações físicas e morais do indivíduo e da sociedade são táticas da racionalidade médica para assegurar a inserção de ambos a uma série de modelos específicos de seu campo de ação.

No âmbito dessa medicalização do social, o saber psiquiátrico vem definindo novas rotulações diagnósticas e novas formas de tratamento do sofrimento psíquico, cunhando termos e definições terapêuticas que se tornaram parte da linguagem cotidiana. Observa-se, assim, um processo de psiquiatrização da vida social, que vem transformando todo o mal-estar psíquico em doença, fato correlato a uma grande valorização da concepção biológica do sofrimento psíquico que, fundamentado na neurologia e na genética, incentivam o tratamento baseado essencialmente em recursos químicos. Essa redução organicista da complexidade dos fatores envolvidos na Saúde Mental tem sido apresentada, pelos adeptos do determinismo biológico, como o principal argumento de uma suposta cientificidade da psiquiatria contemporânea:

A euforia associada às neurociências e à biologia é correlativa de uma marcante biologização do homem. Para os defensores mais radicais do biologicismo que se instalou no pensamento moderno, a natureza humana se reduz à sua estrutura biológica, o mal-estar que a afeta é explicável biologicamente, seu tratamento é biológico, e tudo isto já estaria definitivamente comprovado pela ciência (Bogochvol, 2001, p. 37).

Conforme Birman (2000), “o desenvolvimento da psicofarmacologia e da neurologia no século XX, marcadamente com a descoberta da clorpromazina em 1952, teria alavancado a medicina psiquiátrica a pleitear, finalmente, sua suposta legitimidade científica: a psiquiatria realizou seu sonho, perseguido desde o século XIX, de ser uma especialidade médica, de fato e de direito. Além disso, a psiquiatria ainda poderia se gabar de ter seus fundamentos no discurso rigoroso da ciência biológica." (p. 241).

Assim, atualizando os velhos sonhos organicistas morelianos já plantados na segunda metade do século XIX (Rocha, 1997), a psiquiatria se tornaria um saber fundado, essencialmente, na ciência biológica e, com isso, passaria a definir um novo modo de funcionamento de sua clínica, onde os medicamentos psicofármacos seriam o novo e o principal 
dispositivo para o tratamento e cura dos agora chamados transtornos mentais (Aguiar, 2003).

Essa tendência prescritiva tem convertido os psicofármacos, na expressão de Ferraz (2002), em verdadeiros best sellers do mercado farmacêutico mundial. $\mathrm{Na}$ aliança entre indústria farmacêutica e medicina ocorre um esforço "para estabilizar na sociedade um discurso biológico e o conceito das doenças, fazendo com que a população aprenda a reconhecer em suas experiências de vida os critérios de diagnóstico..." (Aguiar, 2003, p. 8). Essa circunstância tem sido fortemente fomentada pela frenética corrida tecnológica da indústria farmacêutica, que constantemente oferece novas drogas de maior tecnologia e, supostamente, de melhor eficácia do que as anteriormente disponíveis no mercado. Esses novos medicamentos serviriam, em alguns casos, para criar novas indicações terapêuticas ao organizar sintomas antes "dispersos e inespecíficos" em novos quadros nosográficos, para os quais o novo fármaco é indicado como tratamento (Angel, 2007; Cordeiro, 1985). "Na realidade, longe de representar uma forma de intervenção técnica, o novo medicamento substitui o diálogo do médico com o paciente, garante o prestígio profissional e medicaliza problemas vitais" (Cordeiro, 1985, p. 72).

A conjunção de interesses envolvidos na relação entre a indústria farmacêutica e o profissional de medicina, muitas vezes, guarda pouca ou nenhuma relação com a saúde do paciente. O médico ganha credibilidade ao prescrever medicamentos de última geração e, ainda, é recompensado por sua fidelidade através de uma série de vantagens cujo significado financeiro não é nada desprezível (Greenland, 2009). Em contrapartida, o laboratório ganha um engajado representante. Nesse sentido, Telles (2000) apresenta elementos do que chama de "espúrio conluio" formado entre a medicina e a indústria farmacêutica:

(...) a prestigiadíssima New England Journal of Medicine confessou ter por várias vezes cedido a essas pressões [da indústria farmacêutica], publicando artigos, favoráveis a determinadas medicações, escritos por médicos que tinham vínculos financeiros com os laboratórios que fabricavam tais medicações. Isso mostra a que níveis podem chegar essa aliança, envolvendo o que seria o grupo de elite da medicina americana, estabelecendo espúrios conluios entre a indústria farmacêutica e a academia, tudo sacramentado sob o título de "investigações científicas" (s/p).

Circunstanciado por esses fatores, a prescrição de medicamentos, esse procedimento exclusivo da medicina, vem constituindo-se em um avalizador importante do papel de gerenciamento da Saúde Mental exercido pela psiquiatria que, na grande maioria dos casos, tem apresentado a prescrição de medicamentos como a única forma científica de tratamento, mesmo que, para isso, seja necessário passar por cima de qualquer questão que possa implicar uma outra explicação e/ou qualquer outro tipo de atenção que possa ser oferecido ao atendimento do sofrimento psíquico (Amarante, 2007; Barbosa \& Mendes, 2005; Barros, 2008; Ignácio \& Nardi, 2007; Lamb, 2008).

Isto posto, tendo em vista a grande relevância atual da prescrição de psicofármacos no processo de medicalização, realizamos um estudo exploratório sobre a expansão da prescrição de psicofármacos na Rede Pública de Saúde, cujos parâmetros e procedimentos serão apresentados a seguir.

A cidade onde realizamos a pesquisa situa-se na VIII DRS (Departamento Regional de Saúde), região que compreende 25 municípios do sudoeste paulista. Segundo Lamb (2008), essa região apresentou, entre os anos de 2002 e 2006, um aumento de $280 \%$ na administração de psicofármacos dispensados à população pela Rede Pública de Saúde.

Atentos a essa expansão da prescrição de psicofárma$\cos$ na região, o objetivo geral de nossa pesquisa foi o de conhecer, por meio do acompanhamento do registro de prontuários, a trajetória percorrida pela população de usuários do Ambulatório de Saúde Mental de um município de pequeno porte dessa região paulista, desde sua condição de entrada no Pronto Atendimento até a definição do tipo de procedimento terapêutico adotado, com especial atenção aos fatores determinantes da prescrição de psicofármacos. Dentro desse objetivo geral, definimos nossos objetivos específicos como: a) examinar a distribuição percentual dos usuários que já chegam ao serviço de Pronto Atendimento do Ambulatório de Saúde Mental sob prescrição e uso de psicofármacos e investigar a procedência dessa prescrição, b) verificar a distribuição percentual dos encaminhamentos dados aos usuários pelos profissionais do atendimento de entrada do serviço (somente acolhimento, encaminhamento à psicoterapia, encaminhamento à consulta psiquiátrica, encaminhamento simultâneo à psicoterapia e à consulta psiquiátrica, e encaminhamentos a outros médicos da rede pública de saúde), c) investigar a relação entre a prescrição de medicamentos e o tipo de encaminhamento dispensado pelos profissionais do Pronto Atendimento, d) levantar a terapêutica determinada aos usuários encaminhados para tratamento psiquiátrico no Ambulatório e verificar sua relação com a condição de entrada, e) investigar a evolução dos casos de usuários que receberam prescrição de psicofármacos no atendimento psiquiátrico do Ambulatório de Saúde Mental.

\section{Método}

\section{Amostra}

No total, foram examinados 345 prontuários, amostra aleatória estratificada por ano, obtida através de sorteio com o auxílio do programa Bioestat (Ayres, 2007), composta por $20 \%$ dos prontuários do total de usuários que deram entrada no PA no período.

\section{Considerações éticas}

Os procedimentos desta pesquisa foram aprovados pelo Comitê de Ética da Faculdade de Ciências e Letras de Assis/ UNESP (protocolo n 032/2007). 


\section{Procedimentos}

Coleta de dados

A trajetória dos usuários foi estudada a partir da análise das anotações em prontuários dos usuários do serviço de Pronto Atendimento (PA) do Ambulatório de Saúde Mental de uma cidade de pequeno porte do interior paulista no período de 2005 a 2007. O recorte temporal deve-se ao fato de terse iniciado, em fins de 2004, o funcionamento de um Centro de Atenção Psicossocial (CAPS I) no município, serviço no qual passaram a ser atendidos os usuários com quadros diagnósticos considerados mais graves. $\mathrm{O}$ funcionamento desse serviço, desde então, estabeleceu o atual formato da rede de assistência em Saúde Mental naquele município.

\section{Análise dos dados}

Da amostra, foram definidos, conforme os dados dos prontuários, os grupos de usuários que: a) deram entrada ao PA já sob prescrição de psicofármacos, b) deram entrada ao PA sem prescrição psicofarmacológica prévia, c) PA encaminhou à psicoterapia, d) não receberam outro encaminhamento além do PA, e) PA encaminhou à consulta psiquiátrica, f) PA encaminhou simultaneamente à psicoterapia e à consulta psiquiátrica, g) receberam prescrição de psicofármacos quando encaminhados à psiquiatria, h) não receberam prescrição de psicofármacos quando encaminhados à psiquiatria.

O plano de análise da pesquisa quantitativa consistiu na análise exploratória descritiva dos grupos acima caracterizados. Foram calculadas as freqüências percentuais relativas e realizados os cruzamentos e comparações concernentes aos objetivos do estudo.

\section{Resultados}

Sobre a condição de entrada: apresentação de dados sobre o uso prévio de medicação pelos usuários que procuraram o serviço de Pronto Atendimento (PA)

Pesquisamos a condição de entrada, quanto ao uso prévio de medicação, dos sujeitos que foram atendidos no PA ao longo do período delimitado. Fizemos a distribuição dos usuários entre as categorias: usuários que procuraram o PA já com medicação prévia (CMP) e que procuraram o PA sem medicação prévia (SMP). Os resultados dessa distribuição estão apresentados na Tabela 1.

Tabela 1

Distribuição frequencial e percentual dos usuários que deram entrada ao PA, no periodo de 2005 a 2007, segundo sua condição de entrada

\begin{tabular}{lcc}
\hline \multirow{2}{*}{ Condição de entrada } & \multicolumn{2}{c}{ Frequência } \\
\cline { 2 - 3 } & $\mathbf{N}$ & $\mathbf{\%}$ \\
\hline CMP & 224 & 65 \\
SMP & 121 & 35 \\
Total & 345 & 100 \\
\hline
\end{tabular}

CMP: usuários com medicação prévia

SMP: usuários sem medicação prévia
Como mostra a Tabela 1, do exame de 345 prontuários de usuários do ambulatório, constatamos que quase dois terços dos usuários $(65 \%)$ já deram entrada no PA sob prescrição de psicofármacos.

Como a maioria dos usuários chega ao PA já sob prescrição de psicofármacos, investigamos a procedência dessa prescrição prévia aos usuários que deram entrada no PA para saber se provinham, ou não, de prescrição psiquiátrica. Verificamos, então, que apenas $38 \%$ dos usuários previamente medicados receberam sua prescrição psicofarmacológica de médicos psiquiatras. A maioria, $62 \%$ dos casos da amostra, recebeu a prescrição prévia de psicofármacos de outras especialidades médicas, dentre as quais $30 \%$ de clínicos gerais, $20 \%$ de neurologistas, $5 \%$ de ginecologistas e $7 \%$ de outras especialidades.

\section{Sobre os encaminhamentos dispensados aos usuários pelos profissionais do $\mathrm{PA}$}

Conforme os objetivos desta pesquisa, investigamos a proporção dos encaminhamentos dados aos usuários pelos profissionais do Pronto Atendimento (PA). Agrupamos os variados encaminhamentos em cinco categorias: somente PA; encaminhamento à psicoterapia; encaminhamento à consulta psiquiátrica; encaminhamento simultâneo à psicoterapia e consulta psiquiátrica; e encaminhamentos para outros médicos da rede pública de saúde.

Dos 345 usuários que deram entrada no PA, apenas seis não retornaram ao ambulatório e, conseqüentemente, não receberam qualquer encaminhamento. Já excluídos esses casos, a Tabela 2 apresenta a distribuição dos usuários pelas categorias de encaminhamentos acima elencadas.

Tabela 2

Distribuição frequencial e percentual dos encaminhamentos dados aos usuários pelo PA, no período de 2005 a 2007

\begin{tabular}{lcc}
\hline \multirow{2}{*}{\multicolumn{1}{c}{ Encaminhamentos }} & \multicolumn{2}{c}{ Frequência } \\
\cline { 2 - 3 } & N & \% \\
\hline Psicoterapia & 51 & 15 \\
Consulta psiquiátrica & 124 & 37 \\
Psiquiatria e Psicoterapia & 117 & 34 \\
Só Pronto Atendimento & 30 & 9 \\
Outros encaminhamentos* & 17 & 5 \\
Total & 339 & 100 \\
\hline
\end{tabular}

*“Outros encaminhamentos”, no caso desta tabela, refere-se a usuários que foram encaminhados para outros médicos especialistas da rede pública de saúde, no ano de 2007, período em que não havia psiquiatra no serviço.

Como mostra a Tabela 2, somados os usuários que foram encaminhados somente à consulta psiquiátrica aos que foram encaminhados simultaneamente à consulta psiquiátrica e à psicoterapia, temos que $71 \%$ dos usuários receberam encaminhamento psiquiátrico. A porcentagem de encaminhados à psiquiatria se torna ainda maior se levarmos em consideração que os $5 \%$ dos usuários que receberam “outros 
encaminhamentos" foram todos conduzidos a serviços que substituíam o atendimento psiquiátrico provisoriamente faltante em parte do ano de 2007. Temos então que, somadas todas as formas de indicação à psiquiatria, a expressiva maioria de $76 \%$ dos usuários do serviço recebeu encaminhamento ao atendimento psiquiátrico no período estudado. Observamos que o encaminhamento ao atendimento psiquiátrico guarda forte relação com a condição de entrada dos usuários. Realizamos, então, a distribuição desses encaminhamentos conforme a condição de entrada.

\section{Sobre os encaminhamentos dados aos usuários conforme sua condição de entrada}

Investigamos a relação entre a prescrição prévia de medicamentos psicofarmacológicos e o tipo de encaminhamento dado pelos profissionais do PA. Distribuímos os encaminhamentos conforme a sua condição de entrada: com medicação prévia (CMP) e sem medicação prévia (SMP).

Examinando a Tabela 3, percebemos que, no caso do grupo que já chegou sob medicação prévia (CMP), a soma dos atendimentos que compreenderam assistência psiquiátrica, com ou sem o acompanhamento de psicoterapia, resulta em $89 \%$. O percentual de encaminhamentos psiquiátricos chega a $94 \%$ se somarmos a categoria "outros encaminhamentos", composta de usuários que sofreram encaminhamentos substitutivos ao atendimento psiquiátrico faltante no período. Temos então que, do grupo de usuários previamente medicados, um número muito próximo da totalidade, 94\%, recebeu encaminhamento psiquiátrico.

Tabela 3

Distribuição frequencial e percentual dos encaminhamentos dados pelo PA aos usuários segundo a condição de entrada, no período de 2005 a 2007

\begin{tabular}{|c|c|c|c|c|c|c|}
\hline \multicolumn{7}{|c|}{ Condição de entrada } \\
\hline \multirow{2}{*}{ Encaminhamentos } & \multicolumn{2}{|c|}{ Grupo CMP 65\% } & \multicolumn{2}{|c|}{ Grupo SMP 35\% } & \multicolumn{2}{|c|}{ Total } \\
\hline & $\mathbf{N}$ & $\%$ & $\mathbf{N}$ & $\%$ & $\mathbf{N}$ & $\%$ \\
\hline Psicoterapia & 8 & 4 & 43 & 36 & 51 & 15 \\
\hline Psiquiatria & 107 & 49 & 17 & 14 & 124 & 37 \\
\hline Psiquiatria e Psicoterapia & 87 & 40 & 30 & 25 & 117 & 34 \\
\hline Só Pronto Atendimento & 6 & 3 & 24 & 20 & 30 & 9 \\
\hline Outros encaminhamentos* & 11 & 5 & 6 & 5 & 17 & 5 \\
\hline Total & 219 & $100 * *$ & 120 & 100 & 339 & 100 \\
\hline
\end{tabular}

*Outros encaminhamentos, usuários que foram encaminhados para outras especialidades médicas da rede pública de saúde, no ano de 2007.

** A soma das porcentagens, devido aos regulares critérios de aproximação, supera ligeiramente os $100 \%$.

Já no grupo de usuários que se apresentaram sem medicação prévia (SMP), a distribuição dos encaminhamentos é bem diferente: neles, a soma de todas as modalidades de encaminhamentos à psiquiatria resulta em um total de $44 \%$, percentual bem diverso dos $94 \%$ dos encaminhamentos à psiquiatria do grupo previamente medicado.

Examinamos, então, o tratamento dado aos usuários encaminhados à psiquiatria, subdivididos conforme sua condição de entrada.

\section{Sobre o tratamento dado aos usuários que foram encaminhados à psiquiatria}

Investigamos qual foi o tratamento determinado aos usuários que foram encaminhados à consulta psiquiátrica pelos profissionais do PA. Classificamos os tratamentos dados conforme as categorias: tratamento com prescrição de medicação e tratamento sem prescrição de psicofármacos, subdividindo-os conforme a condição de entrada. Os dados, já descontados os 13 casos de usuários que não compareceram à consulta agendada, estão apresentados na Tabela 4.

Tabela 4

Distribuição freqüencial e percentual dos usuários encaminhados à psiquiatria conforme a natureza do tratamento oferecido (medicamentoso ou não medicamentoso), segundo a condição de entrada (CMP ou SMP), no período de 2005 a 2007

\begin{tabular}{|c|c|c|c|c|c|c|}
\hline \multicolumn{7}{|c|}{ Condição de entrada } \\
\hline & \multicolumn{2}{|c|}{ Grupo CMP 65\% } & \multicolumn{2}{|c|}{ Grupo SMP 35\% } & \multicolumn{2}{|c|}{ Total } \\
\hline & $\mathbf{N}$ & $\%$ & $\mathbf{N}$ & $\%$ & $\mathbf{N}$ & $\%$ \\
\hline Medicamentoso & 183 & 99 & 42 & 98 & 225 & 99 \\
\hline Não medicamentoso & 2 & 1 & 1 & 2 & 3 & 1 \\
\hline Total & 185 & 100 & 43 & 100 & 228 & 100 \\
\hline
\end{tabular}

*Dos 241 usuários encaminhados à psiquiátrica, 13 faltaram à consulta. 
Como indica a Tabela 4, dos usuários que compareceram à consulta psiquiátrica, a despeito das mais variadas queixas e sintomas registrados nos prontuários, praticamente todos (99\%), salvo raras e precisas exceções, receberam prescrição psicofarmacológica. Investigamos o motivo específico daqueles três usuários não terem recebido prescrição psicofarmacológica. Pudemos observar que eram casos extremamente excepcionais: uma mulher grávida que não poderia ser medicada com psicofármacos, uma pessoa que apenas solicitava atestado médico-psiquiátrico para constar em requerimento de benefício financeiro ao INSS e um usuário encaminhado pelo Hospital Geral local por tentativa de suicídio relacionada a especificidades da medicação, que foi mantido, então, sob observação sem o uso de medicamentos.

Procuramos, então, investigar a evolução dos casos dos usuários medicados pela psiquiatria.

\section{Sobre a evolução dos casos de usuários medicados pela psiquiatria}

Examinamos os registros de evolução dos casos dos usuários que receberam prescrição de psicofármacos no atendimento psiquiátrico até outubro de 2008, quando foi realizada a coleta dos dados, de forma que compreendem um período variável de dez a quarenta e seis meses, tomando por limites, respectivamente, os casos de final de 2007 e início de 2005. Distribuímos os dados sobre a evolução dos casos nas seguintes categorias: alta no tratamento psiquiátrico, continuação do tratamento psiquiátrico, internação psiquiátrica, encaminhamento ao CAPS para continuidade do tratamento, encaminhamento para tratamento neurológico, encaminhamento a outras especialidades médicas da rede pública de saúde, transferência do tratamento psiquiátrico para outra instituição e abandono do tratamento psiquiátrico.

Dos 225 prontuários de usuários medicados pela psiquiatria, oito não continham anotações referentes à evolução dos casos e, portanto, não constam da distribuição. Feita a distribuição dos 217 casos restantes pelas categorias acima elencadas, obtivemos a Tabela 5.

Tabela 5

Distribuição frequencial e percentual dos usuários medicados pela psiquiatria conforme a evolução dos casos, no período de 2005 a 2007

\begin{tabular}{lcc}
\hline \multicolumn{1}{c}{ Evolução dos casos } & Freqüência \\
\cline { 2 - 3 } & $\mathbf{N}$ & \% \\
\hline Alta no tratamento psiquiátrico & 3 & 1 \\
Continuação do tratamento psiquiátrico & 102 & 47 \\
Internação psiquiátrica & 7 & 3 \\
Encaminhamento ao CAPS para continuidade do tratamento & 30 & 9 \\
Encaminhamento ao atendimento neurológico & 5 & 2 \\
Encaminhamento a outros médicos da Rede pública de saúde & 17 & 8 \\
Transferência do tratamento psiquiátrico para outra instituição* & 4 & 2 \\
Abandono do tratamento psiquiátrico & 73 & 34 \\
Total & 217 & 100 \\
\hline
\end{tabular}

*Usuários que informaram que iriam transferir o tratamento psiquiátrico para outra instituição devido à mudança de cidade ou preferência por médico particular.

\section{Discussão}

Como mostra a Tabela 5, dentre os usuários que foram medicados pela psiquiatria, constatamos que apenas três receberam alta do tratamento psiquiátrico. Procuramos colher mais informações dessas exceções e verificamos que em todos esses casos havia registro de alta por solicitação do próprio usuário. De forma que, salvo essas altas a pedido, não se encontra registro de nenhum caso de usuário que tenha recebido alta do tratamento psiquiátrico medicamentoso. A maioria relativa dos usuários, 47\%, ainda estava sob tratamento psiquiátrico medicamentoso quando da coleta dos dados e $33 \%$ dos usuários haviam abandonado o tratamento psiquiátrico.
A discussão dos nossos dados está apresentada em quatro eixos básicos, conforme a seguinte especificação: a prescrição prévia de psicofármacos, o encaminhamento dado aos usuários pelo serviço de PA, o tratamento dispensado aos usuários encaminhados à psiquiatria e a evolução dos casos medicados pela psiquiatria.

\section{Sobre a prescrição prévia de psicofármacos}

Nossos dados mostram que uma maioria de 65\% dos usuários já chega ao PA sob medicação psicofarmacológica 
e que $62 \%$ desses pré-medicados receberam prescrições de atendimento médico não psiquiátrico. Essa freqüência de prescrições de psicofármacos operada fora das especialidades médicas psiquiátrica e neurológica está em conformidade com a literatura sobre a questão (Almeida, Coutinho, \& Pepe, 1994; Andrade, Andrade, \& Santos, 2004; Falcão, Monsanto, Nunes, Marau, \& Falcão, 2007; Mari \& Jorge, 1997; Noto e cols., 2002). Ainda que se reconheça que medicações psiquiátricas podem ser usadas por outras modalidades clínicas, a prática generalista de prescrição de psicofármacos tem sido associada, pela literatura especializada (Birman, 2000; Roudinesco, 2000), ao incremento da medicalização da população em detrimento de atendimentos mais atentos às condições particulares da pessoa sob sofrimento psíquico:

Diante de qualquer angústia, tristeza ou desconforto psíquico, os clínicos passaram a prescrever, sem pestanejar, os psicofármacos mágicos, isto é, os ansiolíticos e antidepressivos. A escuta da existência e da história dos enfermos foi sendo progressivamente descartada e até mesmo, no limite, silenciada. Enfim, por essa via tecnológica, a população passou a ser ativamente medicalizada, numa escala sem precedentes (Birman, 2000, p. 242).

\section{Sobre o encaminhamento dado aos usuários}

Os dados colhidos são indicativos da existência de uma tendência dos profissionais do PA em encaminharem a ampla maioria dos usuários que dão entrada no ambulatório para a consulta psiquiátrica, acompanhado ou não de outros procedimentos. Essa alta freqüência de encaminhamentos à psiquiatria é fortemente sugestiva de subordinação do trabalho dos profissionais da equipe de saúde a um paradigma eminentemente médico de atendimento em Saúde Mental (Yasui \& Costa-Rosa, 2008; Luzio, 2003; Tenório, 2000).

Em nossos dados, a alta freqüência de encaminhados à psiquiatria pelos profissionais do PA parece estar fortemente influenciada pelo fato de grande parte dos usuários, $65 \%$, já se apresentarem ao serviço sob prescrição prévia de psicofármacos. De fato, nesse subgrupo de pacientes pré-medicados a porcentagem de encaminhamentos à psiquiatria chega a quase totalidade (94\%). A princípio, não há o que estranhar no fato do usuário previamente medicado ser encaminhado à psiquiatria, posto que este encaminhamento seria necessário para avaliar a adequação da medicação e, conforme o caso, confirmá-la, fazer as adequações necessárias, ou suprimi-la. No entanto, esse cuidado aparentemente criterioso é fortemente contraditado por nossos dados que mostram, como examinaremos a seguir, que praticamente todos os usuários pré-medicados encaminhados à psiquiatria recebem prescrição de psicofármacos.

\section{Sobre o tratamento dispensado aos usuários encaminhados à psiquiatria}

Nossos dados mostram que 99\% dos usuários que chegam ao serviço de PA previamente medicados recebem uma reiterativa prescrição psiquiátrica de psicofármacos. Não pudemos comparar qualitativamente as medicações prévias com as prescritas pelo atendimento psiquiátrico do Ambulatório. Mas a verdade é que a inserção do usuário no uso de psicofármacos iniciada por qualquer especialidade médica é sempre acompanhada pela prescrição de psicofármacos por parte do serviço psiquiátrico. Dessa forma, ainda que a consistência de cada prescrição específica não tenha sido objeto do presente estudo, pode-se dizer que o atendimento psiquiátrico estudado nunca nega sua chancela, ainda que possa operar eventuais adequações, à prescrição de psicofármacos generalizada entre outras especialidades médicas.

Nossos dados mostram também que, dentre os usuários encaminhados à psiquiatria sem medicação prévia, $98 \%$ também não escapam da prescrição psicofarmacológica. Isso quer dizer que, independentemente da condição de entrada, o encaminhamento à psiquiatria sempre implica prescrição de psicofármacos. É verdade que encontramos, dentre os 228 usuários encaminhados à psiquiatria, três casos em que não houve prescrição de psicofármaco. Mas, como já pudemos mostrar na apresentação dos dados, tratavam-se de casos especiais em que a medicação era absolutamente contraindicada ou tratava-se de mera obtenção burocrática de um atestado médico.

Nesse sentido, nossos dados corroboram a existência de uma tendência generalizada da psiquiatria de prescrição de psicofármacos que a literatura especializada já percebeu nas ações dos serviços em saúde mental (Bezerra, 1987; Yasui \& Costa-Rosa, 2008; Lamb, 2008; Tenório, 2000). Essa tendência vem estabelecendo o psicofármaco como principal, ou mesmo o único, dispositivo da psiquiatria no tratamento de qualquer tipo de sofrimento psíquico (Amarante, 2007; Bezerra, 1987; Birman, 2000; Guarido, 2007; Ignácio \& Nardi, 2007; Lamb, 2008; Rodrigues, 2003).

Essa verdadeira "compulsão" da psiquiatria em prescrever psicofármacos a qualquer paciente que se encontra sob seu âmbito de ação, além de trazer extraordinárias dificuldades para a construção de outras ações terapêuticas (Luzio, 2003), expõe o usuário a um contato com a droga psicofarmacológica, com seus efeitos colaterais e de dependência (Mendonça \& Carvalho, 2005), do qual nem sempre terá condições de se libertar. Nesse sentido, é importante observar como se desenvolve, em nossos dados, a evolução dos casos medicados pela psiquiatria.

\section{Sobre a evolução dos casos medicados pela psiquiatria}

Nossos dados sobre o desenvolvimento dos casos medicados pela psiquiatria mostram que, quando da coleta dos 
dados, $47 \%$ dos usuários continuavam em tratamento psiquiátrico no próprio ambulatório e a porcentagem dos encaminhados para tratamento em outros serviços, especificados na Tabela 5 de nossa apresentação dos dados, somavam 18\% dos casos. As anotações de abandono de tratamento perfizeram $34 \%$ e os registros de alta, apenas $1 \%$ dos casos.

Desses dados, destaca-se a baixíssima freqüência de alta dos casos encaminhados à psiquiatria, o que sugere uma tendência preocupante. A despeito de a psiquiatria moderna operar amplamente com a noção de transtorno, que não pode deixar de supor algo de transitoriedade, a verdade é que nossos dados não registram a ocorrência de altas no tratamento psiquiátrico. Rigorosamente, encontramos três episódios de alta nos 217 casos acompanhados. No entanto, em todos os casos, foram altas concedidas a pedido do usuário, de forma que, num período que variou de dez a 46 meses de acompanhamento, nossa amostra não conseguiu encontrar nenhum caso de alta determinada pelo atendimento psiquiátrico a qualquer usuário que, por alguma vez, tivesse sido submetido a tratamento com drogas psicofarmacológicas. Os usuários atendidos pela psiquiatria permanecem indeterminadamente submetidos à terapêutica psicofarmacológica, resguardados aqueles que abandonam o tratamento. Como não é plausível considerar o abandono de tratamento como "cura", impossível deixar de ver nessa inexistência de alta uma tendência a cronificação que tende a vincular o usuário ao psicofármaco para todo o sempre, com todos os seus desdobramentos relativos aos efeitos colaterais e à dependência química e psicológica, sempre contrários ao bem-estar e a autonomia do sujeito. Como uma paródia da internação manicomial vitalícia de outros tempos, que as conquistas democráticas tornaram dificilmente defensável (Amarante, 1995, 2007; Amarante \& Torre, 2001; Basaglia, 1985; Costa-Rosa, Luzio, \& Yasui, 2003; De Tilio, 2007; Lougon, 2006; Luzio, 2003; Yasui, 2006), estaríamos sujeitos, hoje, a um tipo de manicomialismo químico que também tende a se estender indeterminadamente.

\section{Considerações finais}

Como já estabelecemos no início deste artigo, o objetivo do presente trabalho foi problematizar o atual processo de medicalização generalizada da população e apresentar um estudo exploratório sobre essa tendência no âmbito de um Ambulatório de Saúde Mental de uma pequena cidade do sudoeste paulista. Consideramos necessário fazer registro de nossa clara consciência dos limites das possibilidades de generalização de nossas observações para outros âmbitos do serviço de atendimento em Saúde Mental. Sabemos que nosso estudo foi realizado em uma pequena instituição desse sistema que, seguramente, apresenta grande heterogeneidade. No entanto, dado o município estudado ser de pequeno porte e apresentar um dos menores índices de consumo de psicofármacos da região, consideramos que as observações suscitadas por esses dados não podem ser incomuns em outras unidades de Saúde Mental e podem demonstrar algumas características do fenômeno estudado em um âmbito mais geral e, sem dúvida, mais preocupante.

Ainda que pesem essas reconhecidas limitações, devemos registrar nestas considerações finais que nossos dados são indicativos de que o dispositivo de saúde mental estudado tem uma atuação fortemente promotora do processo de medicalização psicofarmacológica da população de usuários daquela unidade, cujo perfil é bastante comum nos serviços de saúde mental oferecidos à população em geral. As estereotipias e limitações desse modelo médico-centrado, que parece guiar-se pelo lema "psicofármacos para todos e para sempre", se contrapõem às orientações mais modernas e socialmente atentas da atual Política Nacional de Saúde Mental que, apoiada na lei $10.216 / 02$, preconiza uma atenção psicossocial atenta às complexidades e especificidades do sujeito em sofrimento psíquico. Não se pode desconsiderar que limitações e insuficiências na aplicação dessas diretrizes facilitem o desenvolvimento reducionista e pouco responsável que implementa a medicalização sistemática dos usuários desses serviços. Bem a propósito, dados como os nossos sugerem que, a despeito do amplo espaço discursivo dessas diretrizes entre os profissionais de saúde mental, resistências e insuficiências podem fazer com que os resultados efetivos mostrem-se mais próximos de um processo de medicalização generalizada e crônica da população do que da ampliação responsável dos direitos à saúde mental coletiva que a atual Política Nacional de Saúde Mental procura efetivar.

\section{Referências}

Aguiar, A. A. (2003). Entre as ciências da vida e a medicalizaçãodaexistência:Umacartografiadapsiquiatria contemporânea [Versão eletrônica]. Estados Gerais da Psicanálise: Segundo Encontro Mundial. Recuperado em 28 agosto 2007, de http://www.estadosgerais.org/ mundial_rj/download/2d_Aguiar_47130903_port.pdf

Almeida, L. M., Coutinho, E. S. F., \& Pepe, V. L. E. (1994). Consumo de psicofármacos em uma região administrativa do Rio de Janeiro: Ilha do Governador. Cadernos de Saúde Pública, 10, 5-16.

Amarante, P. (1995). Loucos pela vida: A trajetória da reforma psiquiátrica no Brasil. Rio de Janeiro: Fiocruz.

Amarante, P. (2007). Saúde mental e atenção psicossocial. Rio de Janeiro: Fiocruz.

Amarante, P., \& Torre, E. H. G. (2001). Protagonismo e subjetividade: A construção coletiva no campo da saúde mental. Ciência \& Saúde Coletiva, 6, 73-85.

Andrade, M. F., Andrade, R. C. G., \& Santos, V. (2004). Prescrição de psicotrópicos: Avaliação das informações contidas em receitas e notificações. Revista Brasileira de Ciências Farmacêuticas, 40, 471-479. Recuperado em 25 junho 2009, de http://www.scielo.br/pdf/rbcf/v40n4/ v40n4a04.pdf 
Angel, M. (2007). A verdade sobre os laboratórios farmacêuticos. Rio de Janeiro: Record.

Ayres, M. (2007). Bioestat 5.0 [Software]. Recuperado em 15 novembro 2007, de http://biocistron.blogspot. com/2008/07/programa-bioestat-50_7617.html

Barbosa, C. F., \& Mendes, I. J. M. (2005). Concepção de promoção de saúde de psicólogos no serviço público. Paidéia (Ribeirão Preto), 15, 269-276.

Barros, J. A. C. (2008). Nuevas tendencias de la medicalización. Ciência \& Saúde Coletiva, 13(supl.), 579-587. Recuperado em 23 março 2009, de http://www. scielosp.org/pdf/csc/v13s0/a07v13s0.pdf

Basaglia, F. (1985). A instituição negada (H. Jahn, Trad., 3a ed.). Rio de Janeiro: Graal.

Bezerra, B., Jr. (1987). Considerações sobre terapêuticas ambulatoriais em saúde mental. In S. A. Tundis \& N. R. Costa (Orgs.), Cidadania e loucura: Políticas de saúde mental no Brasil (pp. 134-169). Petrópolis, RJ: Abrasco/ Vozes.

Birman, J. (2000). Mal-estar na atualidade: A psicanálise $e$ as novas formas de subjetivação. Rio de Janeiro: Civilização Brasileira.

Bogochvol, A. (2001). Sobre a psicofarmacologia. In M. C. R. Magalhães (Org.), Psicofarmacologia e psicanálise (pp. 35-61). São Paulo: Escuta.

Cordeiro, H. (1985). A indústria da saúde no Brasil. Rio de Janeiro: Graal.

Costa-Rosa, A., Luzio, C. A., \& Yasui, S. (2003). Atenção psicossocial: Rumo a um novo paradigma na saúde mental coletiva. In P. Amarante (Org.), Archivos de saúde mental e atenção psicossocial (pp. 13-44). Rio de Janeiro: Nau.

De Tilio, R. (2007). A querela dos direitos: Loucos, doentes mentais e portadores de transtornos e sofrimentos mentais. Paidéia (Ribeirão Preto), 17, 195-206.

Falcão, I. M., Monsanto, A., Nunes, B., Marau, J., \& Falcão, J. M. (2007). Prescrição de psicofármacos em medicina geral e familiar: Um estudo na Rede Médicos-Sentinela. Revista Portuguesa de Clínica Geral, 23, 17-30. Recuperado em 24 junho 2009, de http:/www.apmcg.pt/ files/54/documentos/20070525183432838518.pdf

Ferraz, F. C. (2002). Normopatia. São Paulo: Casa do Psicólogo.

Foucault, M. (1979). Microfísica do Poder (R. Machado, Trad.). Rio de Janeiro: Graal.

Foucault, M. (2006). O poder psiquiátrico: Curso dado no Collège de France (1973-1974) (E. Brandão, Trad.). São Paulo: Martins Fontes.

França, S. A. M. (1994). Diálogos com as práticas de saúde mental desenvolvidas na rede de saúde pública. Tese de doutorado não publicada, Pontifícia Universidade Católica de São Paulo, São Paulo.

Guarido, R. (2007). A medicalização do sofrimento psíquico: Considerações sobre o discurso psiquiátrico e seus efeitos na Educação. Educação e Pesquisa, 33, 151-161. Recuperado em 21 junho 2008, de http://www.scielo.br/ pdf/ep/v33n1/a10v33n1.pdf
Gentil, V., Zilberman, M., Lobo, D., Henna, E., Moreno, R., \& Gorenstein, C. (2007). Clomipramine-induced mood and perceived performance changes in selected normal individuals. Journal of Clinical Psychopharmacology, 27, 314-315.

Greenland, P.(2009). New policies needed now on interactions between pharmaceutical companies and physicians. Archives Internal of Medicine, 169, 829-831.

Hora, D. M. (2006). Glossário do Grupo de Estudos e Pesquisas História, Sociedade e Educação no Brasil (HISTEDBR). Faculdade de Educação da Unicamp. Recuperado em 06 abril 2009, de http://www.histedbr.fae. unicamp.br/navegando/glossario/verb_c_medicalizacao. htm.

Ignácio, V. T. G., \& Nardi, H. C. (2007). A medicalização como estratégia biopolítica: Um estudo sobre o consumo de psicofármacos no contexto de um pequeno município do Rio Grande do Sul. Psicologia e Sociedade, 19(3), 88-95.

Lamb, I. G. M. (2008). A prescrição de psicofármacos em uma região de saúde do Estado de São Paulo: Análise e reflexão sobre uma prática. Dissertação de mestrado não publicada, Universidade Estadual Paulista, Assis, SP.

Lougon, M. (2006). Psiquiatria institucional: Do hospício à reforma psiquiátrica. Rio de Janeiro: Fiocruz.

Luzio, C. A. (2003). A atenção em saúde mental em municípios de pequeno e médio portes: Ressonâncias da reforma psiquiátrica. Tese de doutorado não publicada, Universidade Estadual de Campinas, Campinas, SP.

Mari, J. J., \& Jorge, M. R. (1997). Transtornos psiquiátricos na clínica geral. Psychiatry On line Brazil [Versão eletrônica]. Recuperado em 24 junho 2009, de http:// priory.com/psych/tpqcm.htm

Mendonça, R. T., \& Carvalho, A. C. D. (2005). O consumo de benzodiazepínicos por mulheres idosas [Versão eletrônica]. SMAD: Revista Eletrônica Saúde Mental Álcool e Drogas, 1(2). Recuperado em 19 agosto 2007, de http://redalyc.uaemex.mx/pdf/803/80310208.pdf

Noto, A. R., Carlini, E. A., Mastroianni, P. C., Alves, V. C., Galduróz, J. C. F., Kuroiwa, W., Csizmar, J., Csizmar, J., Costa, A., Faria, M. A., Hidalgo, S. R., Assis, D., \& Nappo, S. A. (2002). Análise da prescrição e dispensação de medicamentos psicotrópicos em dois municípios do Estado de São Paulo. Revista Brasileira de Psiquiatria, 24, 68-73.

Rocha, L. C. (1997). Há algo de degenerado no reino da sociedade industrial moderna. In A. Merisse, J. S. Justo, L. C. Rocha, \& M. S. Vasconcelos. Lugares da infância: Reflexões sobre a história da criança na fábrica, creche e orfanato (pp. 11-24). São Paulo: Arte e Ciência.

Rodrigues, J. T. (2003). A medicação como única resposta: Uma miragem do contemporâneo. Psicologia em Estudo, $8(1), 13-22$.

Roudinesco, E. (2000). Por que a psicanálise? (V. Ribeiro, Trad.). Rio de Janeiro: Jorge Zahar. 
Telles, J. S. S. (2000). Psicanálise em debate. Psychiatry On line Brazil, 5(3). Recuperado em 21 maio 2007, de http:// priory.com/psych/psi0300.html

Tenório, F. (2000). Desmedicalizar e subjetivar: A especificidade da clínica da recepção. Cadernos IPUB, 6(17), 79-91.

Yasui, S. (2006). Rupturas e encontros: Desafios da Reforma Psiquiátrica Brasileira. Tese de doutorado não publicada, Escola Nacional de Saúde Pública, Rio de Janeiro.

Yasui, S., \& Costa-Rosa, A. (2008). A estratégia atenção psicossocial: Desafio na prática dos novos dispositivos de Saúde Mental. Saúde em Debate, 32, 27-37.

Daniele de Andrade Ferrazza é doutoranda em Psicologia pelo Programa de Pós-graduação da Faculdade de Ciências e Letras UNESP/Assis da Universidade Estadual Paulista Júlio de Mesquita Filho, campus Assis-SP.

Cristina Amélia Luzio é Professora Assistente Doutora da Faculdade de Ciências e Letras UNESP/Assis da Universidade Estadual Paulista Júlio de Mesquita Filho, campus Assis-SP.

Luiz Carlos da Rocha é Professor Assistente Doutor da Faculdade de Ciências e Letras UNESP/Assis da Universidade Estadual Paulista Júlio de Mesquita Filho, campus Assis-SP.

Raphael Rodrigues Sanches é Mestre em Psicologia pelo Programa de Pós-graduação da Faculdade de Ciências e Letras UNESP/Assis da Universidade Estadual Paulista Júlio de Mesquita Filho, campus Assis-SP.

Recebido: 13/08/2009

$1^{a}$ revisão: $11 / 02 / 2010$

Aceite final: 30/03/2010 